\title{
BMJ Open Exposure to persistent organic pollutants and thyroid cancer risk: a study protocol of systematic review and meta-analysis
}

\author{
Yu Xue Zhang, ${ }^{1}$ Yu Peng Liu, ${ }^{2}$ Su Sheng Miao, ${ }^{3}$ Xiao Dong Liu (D),${ }^{4}$ Shu Mei Ma, ${ }^{4}$ \\ Zhang Yi Qu (iD ${ }^{5}$
}

To cite: Zhang YX, Liu YP, Miao SS, et al. Exposure to persistent organic pollutants and thyroid cancer risk: a study protocol of systematic review and meta-analysis. BMJ Open 2021;11:e048451. doi:10.1136/ bmjopen-2020-048451

- Prepublication history and additional online supplemental material for this paper are available online. To view these files, please visit the journal online (http://dx.doi.org/10. 1136/bmjopen-2020-048451).

YXZ and YPL contributed equally.

Received 26 December 2020 Accepted 05 July 2021

Check for updates

(C) Author(s) (or their employer(s)) 2021. Re-use permitted under CC BY-NC. No commercial re-use. See rights and permissions. Published by BMJ.

For numbered affiliations see end of article.

Correspondence to Professor Zhang Yi Qu; quzy_hmu@163.com

\section{ABSTRACT}

Introduction The thyroid cancer incidence has been increasing all over the world. However, the aetiology of thyroid cancer remains unclear. A growing body of evidence suggested exposure to persistent organic pollutants (POPs) may play a role in the initiation of thyroid cancer, but the results are generally inconsistent across studies. This review aims to synthesise the evidence for the health effects of POPs on the risk of thyroid cancer. Methods and analysis This protocol was reported in accordance to the Preferred Reporting Items for Systematic Reviews and Meta-Analyses Protocols (PRISMA) statements. A comprehensive search, including electronic database search (eg, PubMed, Embase, ProQuest and CNKI), website search and manual search, will be performed to identify all eligible studies. The Population, Exposure, Comparator and Outcome framework was used to clarify the inclusion and exclusion criteria. The Newcastle-0ttawa Scale will be used to assess the quality of included studies. Maximally adjusted effect estimates from individual studies will be summarised with randomeffect models in a conservative manner. $P$ statistics and $Q$-tests will be used to test the heterogeneity across studies. We will perform extensive sensitivity analyses, such as confounding risk ratio (confounding), E-value, fixed-effect models, excluding the most relatively weighted study, including only the high-quality studies and many predesigned subgroup analyses, etc. The findings will be reported in accordance to the PRISMA guidelines.

Ethics and dissemination Ethical approval is not required in this systematic review of published literatures. The results will be published in a peer-reviewed journal and presented at relevant conferences.

PROSPERO registration number CRD42020181343.

\section{INTRODUCTION}

In the last decades, thyroid cancer was the most rapidly increasing cancer worldwide. ${ }^{12}$ In 2020, there were 448915 new cases in women worldwide, representing the fifth-most common cancer in women, compared with 137287 new cases in men, representing the 16 th most common cancer in men. ${ }^{13}$ In the USA, age-adjusted thyroid cancer incidence
Strengths and limitations of this study

- This will be the most comprehensive and up-to-date systematic review to synthesise the evidence on the health effects of exposure to any types of persistent organic pollutants on the incidence risk of thyroid cancer.

- We will use rigorous statistical methods to summarise all the currently eligible data from epidemiological studies, perform extensive sensitivity meta-analyses to evaluate the robustness of our findings.

- The main possible limitations of this study are a limited number of eligible studies and possibly significant heterogeneity between studies.

- Another potential limitation is that it may be difficult to evaluate publication bias if there are not sufficient studies included.

rate have increased approximately fourfold from 4.8 cases/100 000 in 1975 to $17.4 / 100$ 000 in 2020 such that thyroid cancer incidence has been increased at an annual growth rate of about $3 \% .^{34}$ This significant increase pattern was not only unique to the USA but also to a lot of other countries. The growth rate in China was even higher than that in the USA. ${ }^{5}$ In China, the estimated incident cases of thyroid cancer reached 221093 in 2020, accounting for about $38 \%$ of all annually diagnosed thyroid cancer cases. ${ }^{3}$

Although much of the increasing incidence of thyroid cancer is attributed to the improved detection and screening methods, this is unlikely to be the sole cause, since incidence rates are also obviously increasing among children, adolescents and young adults, or for more easily detectable cases with larger tumours. ${ }^{67}$ Therefore, it is urgent to elucidate the aetiology of thyroid cancer. There are only a few well-established risk factors for thyroid cancer: gender, hereditary conditions 
and ionising radiation exposure (particularly when this exposure occurs during childhood). ${ }^{12}$ However, the aetiology remains poorly understood. Recently, a growing body of evidence suggested exposure to persistent organic pollutants (POPs) may play a role in the initiation and development of thyroid cancer, but the results are not conclusive and generally inconsistent across studies.

POPs are a broad class of organic chemicals of global concern, which persist in the environment, biomagnify and bioaccumulate through the food chain, can be transported all over the world. ${ }^{8}$ There are three types of POPs most commonly encountered in the environment: (1) organochlorine pesticides, such as dichlorodiphenyltrichloroethane and its metabolites; (2) industrial chemicals, such as polychlorinated biphenyls and flame retardants (FRs) including polybrominated diphenyl ethers, brominated FRs and organophosphate FRs and (3) unintentional by-products of industrial processes such as polychlorinated dibenzo-p-dioxins and polychlorinated dibenzofurans. ${ }^{9-11}$

In our daily lives, POPs can be virtually detected in many products everywhere. Humans are exposed to POPs in various ways: mainly through diet, but also through the air and the skin absorption. ${ }^{12} 13$ For the general population, the most common exposure route is dietary intake of contaminated fatty foods. Due to the bioaccumulation and biomagnification through the food chain, the highest concentrations of POPs can be found in the human body, as the top of the food chain. ${ }^{13}$

A lot of evidence supports for their significant adverse effects on human health and the environment. Human exposure to these chemicals, even to low levels of POPs can result in many negative health effects including elevated cancer risk, endocrine disruption, immune function impairment and reproductive disorders. ${ }^{13} 14$ Recently, several epidemiological studies have shown that POP exposure has potential carcinogenesis properties in thyroid cancer. ${ }^{15-18}$ However, the findings have been inconsistent. There is only one previous meta-analysis by Han et al., which actually focused on the association of pesticides and thyroid cancer risk, but not for all types of POPs. ${ }^{19}$

\section{OBJECTIVES}

We, therefore, propose to conduct this systematic review and meta-analysis of epidemiological studies to comprehensively summarise the evidence for the effects of exposure to any types of POPs on the incidence risk of thyroid cancer in adult populations. The primary aims of this proposed study are to determine if there is an association between exposure to any types of POPs and the incidence risk of thyroid cancer; and to determine which subtypes of POPs exposure are associated with the thyroid cancer risk. The secondary aim will be to determine which individual POP chemicals are associated with the thyroid cancer risk.

\section{METHODS AND ANALYSIS}

This study has been registered in PROSPERO (CRD42020181343) on 2 May 2020. ${ }^{20}$ We will complete the study by 30 December 2021. The present protocol is in accordance to the PRISMA Protocols guidelines. ${ }^{21}$ This is an original research protocol. Any changes or modifications of the methods stated in this protocol will be updated via PROSPERO and reported in the final systematic review itself. We will report the study according to the PRISMA guidelines (PRISMA). ${ }^{22}$

\section{Eligibility criteria}

The Population, Exposure, Comparator and Outcome framework was used to clarify the eligibility criteria. ${ }^{23}$

\section{Types of populations}

We included studies of adult populations ( $\geq 18$ years old), while studies of children ( $<18$ years) were excluded. Studies that detected and provided exposure levels of individual POPs or any subtype of POPs in biological samples were included, while studies that used only occupations to estimate the occupational exposure to POPs were excluded.

\section{Types of exposures}

We will include studies of exposure to any types of POPs in accordance with the Stockholm Convention definition. ${ }^{8}$ The exposure level to POPs could be detected directly with quantitative measurement in biological samples, including blood, urine, thyroid tissues or adipose tissues.

\section{Types of comparators}

The included comparator will be participants with the lowest exposure level of POPs in individual studies. We will include all these comparisons of the higher exposure levels vs the lowest level of POPs exposure. When the highest exposure level was used as the comparator in original studies, we will use the reciprocal method $(1 / \mathrm{x})$ to convert the effect estimates of the lowest vs the highest level. We will exclude all other types of comparators.

\section{Types of outcomes}

We will include studies that define and classify thyroid cancers using the relevant diagnostic codes in International Classification of Diseases-10 (ICD-10) or other versions of the ICD. If studies do not reported the ICD codes but they provide the information on the cancer site, we will also include these studies. All patients should be diagnosed with clinical-pathological confirmation. The following measurements of thyroid cancer cases should be determined as eligible: (1) diagnosis by a physician; (2) medical records; (3) health insurance data and (4) cancer registry data for diagnosis.

We will exclude all other measurements, including selfreported records without pathological diagnosis. Studies focusing on benign thyroid diseases (including thyroid enlargement and thyroid nodules, etc) but not thyroid cancer will be excluded from this systematic review. 
Types of studies

We will include a broad set of epidemiological studies that investigate the effect of exposure to POPs and thyroid cancer risk over any period. Eligible study designs will be cohort studies (both prospective and retrospective cohorts), case-cohort studies, case-control studies (including nested case-control studies, population-based or hospital-based case-control studies). Due to rigorous ethical principles, there is no eligible randomised controlled trial or non-randomised intervention study in the preliminary search. We will exclude all other study designs (eg, non-original studies, cross-sectional studies, case reports, case series, animal model researches, cell line researches and other mechanism researches).

\section{Types of effect measures}

We will include the effect measures of exposure to any individual POPs or any combined subtype of POPs on the risk of developing thyroid cancer, compared with the lowest exposure level in each original research. All relative effect estimates, namely relative risk ratios (RR), OR and HR are included.

If an original study reports effect estimates from two or more alternative models that have been unadjusted or adjusted for different confounders, then we will systematically prioritise the maximally adjusted estimates from models adjusted for more covariates over those from models adjusted for fewer. For example, if a study presents effect estimates from a crude unadjusted model (model A), a model adjusted for gender (model B) and a model adjusted for gender, age, and body mass index (model C), we will then prioritise the estimate from model C.

\section{Information sources and search}

\section{Electronic bibliographic databases}

The following databases will be searched from the database inception to 29 May 2020: PubMed, Embase, ProQuest and CNKI, with no language restrictions. We will use a combination of Medical Subject Headings terms and corresponding free-text terms to search relevant literatures. The full details of search strategy are presented in online supplemental file 1).

\section{Other electronic database and website search}

We will search two additional grey literature databases for potentially eligible studies:

- Grey Literature Report (http://greylit.org/).

- OpenGrey (http://www.opengrey.eu/).

We will also search the websites of two international organisations for eligible datasets:

- WHO (www.who.int).

- International Agency for Research on Cancer (https://www.iarc.fr/).

\section{Handsearching and expert consultation}

We (YXZ, YPL and SSM) will handsearch for potentially eligible studies in:

- Reference list of all original researches, relevant reviews, editorials and letters.
- Study records that have cited the included studies (identified in Web of Science citation database).

\section{Study selection}

All literature records identified in the search will be imported into the Covidence software ${ }^{24}$ and duplicates will be identified and deleted. Afterwards, two authors (YXZ and YPL) will independently screen titles and abstracts, and then read full texts of potentially relevant literatures. Authors will record specific reasons for exclusion in the full-text screening. A third author (SSM) will resolve any discrepancies. The process of study selection will be reported as per PRISMA guidelines.

\section{Data extraction and data items}

Two authors (YXZ and YPL) will independently extract data, and a third author (SSM) will resolve conflicts. The extracted data items will include study characteristics (including authors, publication year, study country, participants age, gender, year of sample collection and outcome), exposure (including types of POPs, detection methods, comparator), study design (including summary of study design, statistical analysis models used and effect estimates), risk of bias (including selection bias, reporting bias, confounding bias). Extracted data will also include information on the subtype of thyroid cancer and pathophysiological characteristics of the subjects, if available. We will use the predesigned standard sheet to extract data (see online supplemental file 2). This data extraction sheet will be trialled until the authors reach convergence and agreement. Data will be entered into and managed with the Microsoft Excel software. To request missing information, the corresponding author will be contacted by emails (maximum of three emails over 4 weeks).

\section{Risk of bias in individual studies}

Two authors (YXZ and YPL) will independently judge the quality and risk of bias for each study with the NewcastleOttawa Scale (NOS). ${ }^{25}$ Any discrepancies will be resolved by discussion or consultation with a third author (XDL). All quality assessors will jointly trial the NOS criteria until they have synchronised their understanding and application of the NOS scale. The NOS scale consists of three domains of bias: selection (four points), comparability (two points) and outcome (three points). According to the sum points of three domains, individual studies will be categorised as either high $(\geq 6)$ or low $(<6)$ quality. We will report the study-level risk of bias assessments by domains in a summary table (online supplemental file 3).

\section{Synthesis of results}

Quantitative synthesis

In the primary meta-analysis, we will use the maximally adjusted effect estimates and corresponding 95\% CIs to summarise the effect of POPs exposure on the risk of thyroid cancer. To be more rigorous and conservative, we will use random-effect models rather than fixed-effect models to summarise the effect sizes. In the primary metaanalysis, we will assess the overall effect of exposure to all 
types of POPs and each subtype of POPs on the thyroid cancer risk. We will also assess the effect of exposure to any individual POPs, if the number of eligible studies is no less than three.

When two or more studies from the same cohort or data source are eligible for inclusion in the meta-analysis, we will prioritise in this order: (1) the study with the highest quality score and the lowest risk of bias; (2) the study with the most informative measurements of exposure to POPs; (3) the study with large sample size; (4) the study with the longest follow-up and (5) the study with the maximum adjustment for relevant potential confounding factors.

When studies only report effect sizes for each individual POP separately, we will initially combine these individual effects into a study-specific overall effect of total or subtype of POPs with fixed-effect models. When studies only report separately effect sizes on each individual type of thyroid cancer, we will also combine these effects into a study-specific overall effect for all types of thyroid cancer with fixed-effect models.

Comprehensive Meta Analysis V.2.2.046 (Biostat, Englewood, New Jersey, USA) will be used for all analyses. Statistical significance was defined as two-sided $p$ values less than 0.05 in the major meta-analysis. In all subgroup analyses, we will use the Bonferroni method to correct the level of statistical significance.

\section{Heterogeneity inspection}

We will calculate the $\mathrm{I}^{2}$ statistic and perform the Cochrane Q-test to test the heterogeneity across studies and will report $\mathrm{I}^{2}$ statistics and $\mathrm{p}$ values in the systematic review. Based on the $\mathrm{I}^{2}$ statistics, an $\mathrm{I}^{2}$ value of $\leq 30 \%$ represents low heterogeneity, $30 \%-50 \%$ moderate heterogeneity, $50 \%-75 \%$ substantial heterogeneity and $>75 \%$ considerable heterogeneity. If there is substantial between-study heterogeneity, we will perform meta-regression and subgroup analyses to explore potential sources of the heterogeneity. Meta-regression analyses will be performed according to sample size, year of blood sample collection or study publication. Subgroup analyses are elaborated in the section of 3.6.4. Sensitivity analysis.

\section{Publication bias}

To assess potential publication bias, we will conduct the Begg's and Egger's tests and further rigorously adjust for the summarised results by applying the Duval and Tweedie's trim and fill method. We will also use funnel plots to ascertain presence of publication bias, if ten or more eligible studies (datasets or comparisons) are included in our systematic review.

\section{Sensitivity analysis}

We will perform a broad set of predesigned sensitivity analyses to evaluate the robustness of the findings, as follows:

- We will conduct a sensitivity analysis using fixed-effect models.
- The minimally adjusted effect estimates will be pooled and these results will be compared with the primary results from the maximally adjusted effects using the Confounding RR method, which is defined as the ratio of pooled results of the maximally adjusted versus minimally adjusted data. ${ }^{26}$ Confounding RRs are used to evaluate whether the underlying confounders controlled in each individual studies could have influenced the results.

- To assess the potential impact of residual confounding bias, we will perform E-value analysis. ${ }^{27} \mathrm{E}$-value shows the minimum strength of association that a hypothetical residual confounding factor would need to have with both the exposure to POPs and the incidence risk of thyroid cancer to fully explain the observed effect.

- A sensitivity analysis by removing the most relatively weighted study (or dataset) will be performed to assess its influence on the results and to explore potential sources of heterogeneity across studies.

- A sensitivity analysis including only the high-quality studies will be performed.

- We will also perform a sensitivity analysis by including only the prospective studies (including cohort studies, case-cohort studies and nested case-control studies), because the prospective nature of these study designs is invaluable for confirming the temporal sequence of POPs exposure and thyroid cancer onset and therefore helps to examine causal associations.

- We will performed predefined subgroup metaanalyses by geographic regions (as per WHO Regions or World Bank Income Country Groups), sample size ( $\geq$ median vs $<$ median), year of blood sample collection (before vs after 2010), gender (female vs male) and age of participants ( $<50 \mathrm{vs} \geq 50$ years), if eligible studies in each subgroup are no less than three. If else, these aforementioned subgroup analyses will be performed as sensitivity analyses by excluding the subgroup with less than three studies.

- We will also perform cumulative meta-analyses according to sample size of each individual study from small to large, year of blood drawing or study publication in chronological order from front to latest.

We will also perform some post hoc subgroup analyses or other additional analyses, whenever feasible.

\section{Quality of evidence assessment}

All authors will together assess quality of evidence for the entire body of evidence by exposures (total POPs or subtypes of POPs). We will use the Grading of Recommendations Assessment, Development and Evaluation approach to grade the quality of evidence as 'high', 'moderate', 'low' or 'very low'. ${ }^{28}$ The GRADEproGDT software online version will be used to summarise the quality of evidence.

We will downgrade or upgrade the quality of evidence according to the following domains: (1) study design; (2) risk of bias; (3) inconsistency; (4) indirectness; (5) imprecision; (6) publication bias and (7) confounding 
bias. Within each domain, we will categorise the concern for the quality of evidence as 'none', 'serious' and 'very serious'. In the meta-analysis, if the prospective epidemiological studies (including cohort, case-cohort and nested case-control studies) accounted for more than $60 \%$ of all included studies, we will start at 'high' for the quality of evidence; or else, we will start at 'moderate'. Afterwards, we will downgrade the quality of evidence for no concern by nil grades $(0)$, for a serious concern by one grade $(-1)$, or for a very serious concern by two grades $(-2)$. We will upgrade the quality by one grade for each of the following reasons: large effect size $(+1)$, a significant dose-response relationship $(+1)$ or plausibility that residual confounders cannot explain the observed effect $(+1)$.

For example, we start at 'moderate' for a body of evidence consisting of 10 studies (including five cohorts and five case-control studies). If there is a serious concern for both risk of bias $(-1)$ and inconsistency $(-1)$, meanwhile the summarised effect size is large $(+1)$; but there is no other concerns and no other upgrading reasons, then we will downgrade the quality by one grade from 'moderate' to 'low'.

\section{Patient and public involvement}

Patients and the public were not involved in the design, conduct, or reporting of our present study.

\section{ETHICS AND DISSEMINATION}

Ethical approval is not required in this systematic review of published literatures. The results will be published in a peer-reviewed journal and presented at relevant conferences to promote knowledge transfer.

\section{Author affiliations}

${ }^{1}$ Department of Preventive Medicine, School of Public Health, Harbin Medical University, Harbin, Heilongjiang, China

${ }^{2}$ Department of Epidemiology and Biostatistics, School of Public Health and Management, Wenzhou Medical University, Wenzhou, China

${ }^{3}$ Department of Head and Neck Surgery, Harbin Medical University Cancer Hospital, Harbin, Heilongjiang, China

${ }^{4}$ Department of Occupational and Environmental Health, School of Public Health and Management, Wenzhou Medical University, Wenzhou, China

${ }^{5}$ Department of Hygiene Microbiology, School of Public Health, Harbin Medical University, Harbin, Heilongjiang, China

Contributors YXZ, YPL, ZYQ and SMM conceived and designed this systematic review. All authors developed the selection criteria, risk bias assessment strategy and data extraction criteria. YXZ, YPL and SSM developed the pilot search strategy. YXZ and YPL will be the two title, abstract and full-text reviewers. SSM or XDL will be the third reviewer that will help resolve any discrepancy. YXZ and YPL wrote the initial draft of the protocol. All authors revised the manuscript critically for important intellectual content. All authors approved the final version of the systematic review to be published: All authors. ZYQ and SSM are the guarantor of the systematic review.

Funding This work was supported by the Natural Science Foundation of Heilongjiang Province (YQ2019H021 to YPL); the China Postdoctoral Science Foundation (2018M641875 to YPL); the Basic Medical and Health Science Project of Wenzhou Science and Technology Bureau (Y20190191 to YPL).

Disclaimer The funders and sponsors had no role in the design and conduct of the study; collection, management, analysis, and interpretation of the data; preparation, review, or approval of the manuscript; and decision to submit the manuscript for publication.
Competing interests None declared.

Patient consent for publication Not required.

Provenance and peer review Not commissioned; externally peer reviewed.

Supplemental material This content has been supplied by the author(s). It has not been vetted by BMJ Publishing Group Limited (BMJ) and may not have been peer-reviewed. Any opinions or recommendations discussed are solely those of the author(s) and are not endorsed by BMJ. BMJ disclaims all liability and responsibility arising from any reliance placed on the content. Where the content includes any translated material, BMJ does not warrant the accuracy and reliability of the translations (including but not limited to local regulations, clinical guidelines, terminology, drug names and drug dosages), and is not responsible for any error and/or omissions arising from translation and adaptation or otherwise.

Open access This is an open access article distributed in accordance with the Creative Commons Attribution Non Commercial (CC BY-NC 4.0) license, which permits others to distribute, remix, adapt, build upon this work non-commercially, and license their derivative works on different terms, provided the original work is properly cited, appropriate credit is given, any changes made indicated, and the use is non-commercial. See: http://creativecommons.org/licenses/by-nc/4.0/.

\section{ORCID iDs}

Xiao Dong Liu http://orcid.org/0000-0002-6879-990X

Zhang Yi Qu http://orcid.org/0000-0002-6703-813X

\section{REFERENCES}

1 Bray F, Ferlay J, Soerjomataram I, et al. Global cancer statistics 2018: GLOBOCAN estimates of incidence and mortality worldwide for 36 cancers in 185 countries. CA Cancer J Clin 2018;68:394-424.

2 American Cancer Society. Thyroid cancer. Available: https://www. cancer.org/cancer/thyroid-cancer.html [Accessed 22 Nov 2020].

3 Ferlay J, Ervik M, Lam F. Global cancer Observatory: cancer today. Lyon, France: international agency for research on cancer. Available: https://gco.iarc.fr/today/home [Accessed 22 Nov 2020].

$4 \mathrm{Lim} \mathrm{H}$, Devesa SS, Sosa JA, et al. Trends in thyroid cancer incidence and mortality in the United States, 1974-2013. JAMA 2017;317:1338-48.

5 Frank W, Dillwyn W. Thyroid cancer. In: Stewart BW, Wild CP, eds. World cancer report 2014. Lyon, France: International Agency for Research on Cancer, WHO Press, 2014: 738-50.

6 Qian ZJ, Jin MC, Meister KD, et al. Pediatric thyroid cancer incidence and mortality trends in the United States, 1973-2013. JAMA Otolaryngol Head Neck Surg 2019;145:617-23.

7 Bernier M-O, Withrow DR, Berrington de Gonzalez A, et al. Trends in pediatric thyroid cancer incidence in the United States, 1998-2013. Cancer 2019;125:2497-505.

8 Stockholm Convention. The POPs. Available: http://www.pops.int/ TheConvention/ThePOPs/tabid/673/Default.aspx [Accessed 22 Nov 2020].

9 European Commision Enviroment Chemicals. Persistant organic pollutants (POPs). Available: https://ec.europa.eu/environment/ chemicals/international_conventions/index_en.htm [Accessed 22 Nov 2020].

10 Guo W, Pan B, Sakkiah S, et al. Persistent organic pollutants in food: contamination sources, health effects and detection methods. Int $J$ Environ Res Public Health 2019;16:4361.

11 United States Environmental Protection Agency. International cooperation. persistent organic pollutants: a global issue, a global response. Available: https://www.epa.gov/international-cooperation/ persistent-organic-pollutants-global-issue-global-response\#pops [Accessed 02 Dec 2020].

12 Abdallah MA-E, Pawar G, Harrad S. Evaluation of in vitro vs. in vivo methods for assessment of dermal absorption of organic flame retardants: a review. Environ Int 2015;74:13-22.

13 World Health Organization. Food safety: persistent organic pollutants (POPs). Available: https://www.who.int/news-room/q-a-detail/foodsafety-persistent-organic-pollutants-(pops) [Accessed 22 Nov 2020].

14 Ashraf MA. Persistent organic pollutants (POPs): a global issue, a global challenge. Environ Sci Pollut Res Int 2017;24:4223-7.

15 Zhang Q, Hu M, Wu H, et al. Plasma polybrominated diphenyl ethers, urinary heavy metals and the risk of thyroid cancer: a case-control study in China. Environ Pollut 2021;269:116162.

16 Huang $\mathrm{H}$, Sjodin A, Chen Y, et al. Polybrominated diphenyl ethers, polybrominated biphenyls, and risk of papillary thyroid cancer: a nested case-control study. Am J Epidemiol 2020;189:120-32. 
17 Han X, Meng L, Li Y, et al. Associations between exposure to persistent organic pollutants and thyroid function in a case-control study of East China. Environ Sci Technol 2019;53:9866-75.

18 Deziel NC, Warren JL, Huang H, et al. Exposure to polychlorinated biphenyls and organochlorine pesticides and thyroid cancer in Connecticut women. Environ Res 2021;192:110333.

19 Han MA, Kim JH, Song HS. Persistent organic pollutants, pesticides, and the risk of thyroid cancer: systematic review and meta-analysis. Eur J Cancer Prev 2019;28:344-9.

20 Liu Y, Zhang Y, Qu Z. Associations between exposure to persistent organic pollutants and the risk of thyroid cancer: a systematic review and meta-analysis. PROSPERO 2020 CRD42020181343, 2020. Available: https://www.crd.york.ac.uk/prospero/display_record.php? ID=CRD42020181343

21 Shamseer L, Moher D, Clarke M, et al. Preferred reporting items for systematic review and meta-analysis protocols (PRISMA-P) 2015: elaboration and explanation. BMJ 2015;349:97647.

22 Liberati A, Altman DG, Tetzlaff J, et al. The PRISMA statement for reporting systematic reviews and meta-analyses of studies that evaluate healthcare interventions: explanation and elaboration. BMJ 2009;339:b2700.

23 Morgan RL, Whaley P, Thayer KA, et al. Identifying the PECO: a framework for formulating good questions to explore the association of environmental and other exposures with health outcomes. Environ Int 2018;121:1027-31.

24 Covidence Community. Covidence, 2020. Available: https:// community.cochrane.org/help/tools-and-software/covidence [Accessed 25 Nov 2020].

25 Wells GA, Shea B, O'Connell D. The Newcastle-Ottawa Scale (NOS) for assessing the quality if nonrandomized studies in meta-analyses. Available: http://www.ohri.ca/programs/clinical_epidemiology/oxford. asp [Accessed 16 Dec 2020].

26 Greenland S, Pearce N. Statistical foundations for model-based adjustments. Annu Rev Public Health 2015;36:89-108.

27 VanderWeele TJ, Ding P. Sensitivity analysis in observational research: introducing the E-Value. Ann Intern Med 2017;167:268-74.

28 Guyatt GH, Oxman AD, Vist GE, et al. Grade: an emerging consensus on rating quality of evidence and strength of recommendations. $B M J$ 2008;336:924-6. 